\title{
Breit-Wigner Formulas for the Scattering Phase and the Total Scattering Cross-Section in the Semi-Classical Limit
}

\author{
C. Gérard ${ }^{1}$, A. Martinez ${ }^{2}$, and D. Robert ${ }^{3}$ \\ 1 Ecole Polytechnique, Centre de Mathématiques, F-91128 Palaiseau Cédex, France \\ 2 Université de Paris-Sud, Département de Mathématiques, Bât 425, F-91405 Orsay Cédex, \\ France \\ 3 Université de Nantes, Département de Mathématiques, 2, chemin de la Houssinière, \\ F-44072 Nantes Cédex 03, France
}

\begin{abstract}
In this paper we prove results in resonance scattering for the Schrödinger operator $P_{V}=-h^{2} \Delta+V, V$ being a smooth, short range potential on $\mathbf{R}^{n}$. More precisely, for energy $\lambda$ near a trapping energy level $\lambda_{0}$ for the classical system defined by the Hamiltonian $p(x, \xi)=\xi^{2}+V(x)$, we prove that the scattering phase and the scattering cross sections associated to $\left(P_{V}, P_{0}\right)$ have the Breit-Wigner form ("Lorentzian line shape") in the limit $h \rightarrow 0$.
\end{abstract}

\section{Introduction}

We consider in this paper the semiclassical asymptotics of the scattering phase $s(\lambda, h)$ and the total scattering cross-section $\sigma(\omega, \lambda, h)$ associated to the Schrödinger operator $P=-h^{2} \Delta+V(x)$ on $\mathbb{R}^{n}$, for $\lambda$ near a trapping energy level $\lambda_{0}$. By this, we mean that the classical flow associated to the Hamiltonian $p(x, \xi)=\xi^{2}+V(x)$ has trapped trajectories in $p^{-1}\left(\lambda_{0}\right)$. Moreover, we will here assume that classical particles are trapped in $p^{-1}\left(\lambda_{0}\right)$ due to the presence of a potential well.

This configuration gives rise to the well known shape resonances for the quantum Hamiltonian, which have been extensively studied in the last few years: see for example the works of Helffer-Sjöstrand [He-Sj], Combes-Duclos-KleinSeiler [C-D-K-S], and Hislop-Sigal [Hi-Si].

On the other hand, the problem of the short wave asymptotics for the scattering phase has been studied by many authors in optical and acoustical scattering problems. Let us mention for instance the works of Jensen-Kato [Je-Ka]; Majda-Ralston [Maj-Ra]; Petkov-Popov [Pe-Po] and Ivrii-Shubin [Iv-Sh].

For the Schrödinger operator, there are works of Colin de Verdière [CdV] and Guillopé $[\mathrm{Gu}$ ] in the high energy limit, and of Robert-Tamura [Ro-Ta] in the semiclassical limit $h \rightarrow 0$.

However, all these results require a kind of non-trapping assumption in order to avoid problems caused by resonances (or poles of the S-matrix) close to the real axis. 
For the scattering cross-section, Robert and Tamura [Ro-Ta1] have studied the semiclassical asymptotics of $\sigma(\omega, \lambda, h)$ under the non-trapping condition. Let us also mention the works of Sobolev-Yafaev [So-Ya], Yajima [Yaj], and EnssSimon [En-Si], where the last three works concern averaged (in $\lambda$ or in $\omega$ ) scattering cross-sections, and hence do not require a condition on the classical flow.

For physical reasons, related for example to time delay, it is believed that resonances close to the real axis should produce big variations in the scattering phase. We refer the interested reader to the book of R. G. Newton [Ne, Chap. 12] for the link between the time delay and the scattering phase. This qualitative behavior is described for instance in the well known Breit-Wigner formulas (cf. [La-Li, Sect. 142]).

In this paper we give a rigorous proof of these formulas for the scattering phase and the scattering cross-section in the semiclassical limit. In particular, we prove that the scattering phase increases by 1 at a shape resonance (see Corollary 2.3). We use the results obtained by two of the authors in [Ge-Ma 1] on the asymptotics of the spectral function of $P$ to prove our result, using also estimates on the resolvents of $P$ in the same spirit as in [Ro-Ta1].

We also want to mention a recent paper of Nakamura [Na] who studies BreitWigner formulas for the scattering amplitude under assumptions very similar to ours.

Let us now recall some results about scattering theory.

We shall always assume that $V \in C^{\infty}\left(\mathbb{R}^{n} ; \mathbb{R}\right)$ is such that, for some $\varrho>0$ :

$$
\left(V_{\varrho}\right) \quad \forall \alpha \in \mathbb{N}^{n}, \quad\left|D_{x}^{\alpha} V(x)\right| \leqq C_{\alpha}\langle x\rangle^{-\varrho-|\alpha|},
$$

where $\langle x\rangle=\left(1+|x|^{2}\right)^{1 / 2}$. Moreover, we shall assume that $V$ is always short-range $(\varrho>1)$ or stronger assumptions necessary to define the scattering phase and the scattering cross-section.

We consider the wave operators:

$$
W_{ \pm}=s-\lim _{t \rightarrow \pm \infty} \exp (- \text { it } P) \cdot \exp \left(\text { it } P_{0}\right)
$$

which exist and are complete under assumption $\left(V_{\varrho}\right)$ with $\varrho>1$. Then, the scattering operator is defined by:

$$
S=W_{-}^{*} W_{+}
$$

and can be decomposed on the spectral representation of $P_{0}=-h^{2} \Delta$ as:

$$
(S f)(\lambda, \omega)=(S(\lambda) f(\lambda, \cdot))(\omega)
$$

for $\lambda>0, \omega \in S^{n-1} \cdot S(\lambda)$ is the scattering matrix and is unitary on $L^{2}\left(S^{n-1}\right)$.

If $\varrho>n$, then $S(\lambda)=1-2 i \pi \cdot T(\lambda)$, where $T(\lambda)$ is a trace class operator. It is then possible to define det $S(\lambda)$ (which is of modulus 1 ), and the scattering phase $s(\lambda, h)$ is defined by:

$$
\operatorname{det} S(\lambda)=e^{2 i \pi s(\lambda, h)} .
$$

(In physics books, the definition $\operatorname{det} S(\lambda)=\mathrm{e}^{2 i s(\lambda, h)}$ is also used.) 
If now we only have $\varrho>\frac{n+1}{2}$, then $T(\lambda)$ is a Hilbert-Schmidt operator. If we denote $T(\theta, \omega, \lambda, h)$ its distribution kernel, then the total scattering cross-section $\sigma(\omega, \lambda, h)$ is defined as:

$$
\sigma(\omega, \lambda, h)=c(\lambda, h) \cdot \int_{S^{n-1}}|T(\theta, \omega, \lambda, h)|^{2} d \theta,
$$

where $c(\lambda, h)=(2 \pi)^{n+1} \lambda^{-(n-1) / 2} h^{n-1}$.

Now. let us denote by $d E_{\lambda}=\frac{\partial E}{\partial \lambda} d \lambda$ the spectral resolution of $P$ for $\lambda>0$, and by $\frac{\partial e}{\partial \lambda}(x, y, \lambda, h)$ the spectral function of $P$, i.e. the distribution kernel of $\frac{\partial E}{\partial \hat{\lambda}}$. Then, from the stationary scattering theory for Schrödinger operators, and the BirmanKrein theory [Bi-Kr] (see for example the book [Re-Si] and [Ro-Ta]), we get the following remarkable formulas:

$$
\begin{gathered}
\frac{d s}{d \lambda}(\lambda, h)=\frac{1}{2 \lambda} \int_{R^{n}}(2 V(x)+x \cdot \nabla V(x)) \frac{\partial e}{\partial \lambda}(x, x, \lambda, h) d x, \\
\sigma(\omega, \lambda, h)=\pi \lambda^{1 / 2} \cdot h^{-1} \int_{R^{n} \times R^{n}} \frac{\partial e}{\partial \lambda}(x, y, \lambda, h) V(x) V(y) e^{i V \bar{\lambda}(x-y) \cdot \omega / h} d x d y .
\end{gathered}
$$

Let us remark here that, since $s(\lambda, h)$ is only defined modulo $\mathbb{Z}, \frac{d s}{d \hat{\lambda}}$ carries all the relevant information on $s(\lambda, h)$. Formulas $(0.1)$ and $(0.2)$ will be essential for proving our results.

The plan of the paper is the following:

- In Sect. I, we prove a global semiclassical estimate on $\frac{\partial e}{\partial \lambda}$.

- In Sect. II, we use this estimate to get a Breit-Wigner formula for $\frac{d s}{d \lambda}$ when $Q>n+1$, and we show that the increases $\Delta_{ \pm} s(h)$ of the phase shift around the real part of a resonance tend to $1 / 2$ as $h$ tends to 0 .

- In Sect. III we give a Breit-Wigner formula for the total scattering crosssection for $\varrho>\frac{n+1}{2}$.

\section{Technical Preparations}

In this section we prove a technical result which will be used in the next section to estimate some remainder terms in the scattering phase.

We consider the semiclassical Schrödinger operator:

$$
P=-h^{2} \Delta+V(x) \text { on } \mathbb{R}^{n},
$$

where $V \in C^{\infty}\left(\mathbb{R}^{n} ; \mathbb{R}\right)$ satisfies:

(H1) $V$ has a potential well in an island, which means:

There exist a connected open set $\hat{O} \Subset \mathbb{R}^{n}$ and a connected compact set $U \Subset \hat{O}$ such that:

$$
V \leqq \lambda_{0} \text { on } U, \quad V>\lambda_{0} \text { on } \hat{O} \backslash U, \quad V<\lambda_{0} \text { on } \mathbb{R}^{n} \backslash \hat{O}
$$


Here $\lambda_{0}>0$ is some fixed energy level.

(H2) $V$ is dilation analytic outside the island $\hat{O}$, i.e.

$V$ has an holomorphic extension to

$\left\{z \in \mathbb{C}^{n}|| \operatorname{Im} z \mid \leqq \varepsilon_{0}\langle\operatorname{Re} z\rangle, \operatorname{Re} z\right.$ is in a neighborhood of $\left.\mathbb{R}^{n} \backslash \hat{O}\right\}$

and satisfies $|V(z)|=\mathscr{O}\left(\langle z\rangle^{-\varrho}\right)$ uniformly in this set, where $\varrho>0, \varepsilon_{0}>0$.

(H3) $\lambda_{0}$ is non-trapping outside the island $\hat{O}$, i.e.:

$$
\begin{gathered}
\forall(x, \xi) \in T^{*} \mathbb{R}^{n} \text { such that } p(x, \xi)=\lambda_{0} \text { and } x \in \mathbb{R}^{n} \backslash \hat{O} \text {, then } \\
\left|\operatorname{expt} H_{p}(x, \xi)\right| \rightarrow \infty \quad \text { when } \quad|t| \rightarrow \infty .
\end{gathered}
$$

Here $p(x, \xi)=\xi^{2}+V(x)$ is the principal symbol of $P$, and $H_{p}$ denotes the hamiltonian field associated to it.

Under assumptions (H1) to (H3), we can define in a neighborhood of $\lambda_{0}$ the set $\Gamma(h)$ of the resonances of $P$, as in [He-Sj] or [Ag-Co] (cf. [He-Ma] for the equivalence between these definitions).

We assume also then:

(H4) There exists a complex open set $\Omega(h)$ with $\Omega(h) \cap \mathbb{R} \neq \emptyset$ shrinking to $\left\{\lambda_{0}\right\}$ as $h$ tends to 0 , such that $\Gamma(h) \cap \Omega(h)$ is a simple point $\{\varrho(h)\}$, with: $\forall \varepsilon>0, \exists C_{\varepsilon}>0$ such that

$$
d(\Gamma(h), \partial \Omega(h)) \geqq \frac{1}{C} e^{-\varepsilon / h} .
$$

In fact, by the results of $[\mathrm{He}-\mathrm{Sj}$, Sect.9], this assumption actually is an assumption on the point spectrum, near $\lambda_{0}$, of the Dirichlet realization of $P$ in a neighborhood of the well $U$ (see also infra).

Now, we denote by $R(\hat{\lambda} \pm i 0, h)$ the usual boundary values of the resolvent $R(z)=(P-z)^{-1}$, obtained by the limiting absorption principle. Recall that $R(\lambda \pm i 0, h)$ are bounded operators from $L_{s}^{2}$ into $L_{-s}^{2}$, where

$$
L_{s}^{2}=L^{2}\left(\langle x\rangle^{2 s} \cdot d x\right), \quad \text { for } s>\frac{1}{2} .
$$

Then, the result of this section is the following one:

Proposition 1.1. Under assumptions (H1) to (H4), and denoting $\Sigma=\mathbb{R}^{n} \backslash \hat{O}$ (the sea), we have:

$\forall \varepsilon>0, \forall s>\frac{1}{2}, \forall \alpha, \beta \in \mathbb{N}^{n}$ with $|\alpha|+|\beta| \leqq 2$ :

$$
\left\|1_{\Sigma}\langle x\rangle^{-s}\left(h \partial_{x}\right)^{\alpha} R(\lambda \pm i 0, h)\left(h \partial_{x}\right)^{\beta}\langle x\rangle^{-s} 1_{\Sigma}\right\|=O\left(h^{-1}\right)+O_{\varepsilon}\left(\frac{e^{-\left(2 S_{0}-\varepsilon\right) / h}}{|\lambda-\varrho(h)|}\right)
$$

uniformly for $h>0$ small enough, and $\lambda \in I(h):=\Omega(h) \cap \mathbb{R}$. Here $S_{0}>0$ denotes the Agmon distance (associated to the metric $\left.\left(V(x)-\hat{i}_{0}\right)_{+} d x^{2}\right)$ between $U$ and $\partial \hat{O}$.

We first prove:

Lemma 1.2. For any compact set $K \subset \sum$ and any $\alpha, \beta \in \mathbf{N}^{n}$ with $|\alpha|+|\beta| \leqq 2$, we have: $\forall \varepsilon>0$,

$$
\left\|1_{K}\left(h \partial_{x}\right)^{\alpha} R(\lambda \pm i 0)\left(h \partial_{x}\right)^{\beta} 1_{K}\right\|=O\left(h^{-1}\right)+O_{\varepsilon}\left(\frac{e^{-\left(2 S_{0}-\varepsilon\right) / h}}{|\lambda-\varrho(h)|}\right)
$$

uniformly for $h>0$ small enough and $\lambda \in I(h)$. 
Proof. Let us first recall a formula from [Ge-Ma 1]: If we let $P$ act on the HelfferSjöstrand space $H\left(\Lambda_{t G},\langle\xi\rangle^{2}\right)$ [where $G(x, \xi)=x \cdot \xi$ for $|x|$ large enough, $\left.p(x, \xi)=\lambda_{0}\right]$, and if we denote by $R_{t}(\lambda)$ its resolvent $(\lambda \in I(h), t \neq 0,|t|$ small enough), then we have for $t>0$ (cf. [Ge-Ma1, formula (3.12]):

$R_{t}(\lambda)=\left(\tilde{P}_{t}-\lambda\right)^{-1}+(\varrho(h)-\lambda)^{-1} E_{t}^{+} E_{t}^{-}+E_{t}(\lambda) W\left(\tilde{P}_{t}-\lambda\right)^{-1}-E_{t}^{+} E_{t}^{-}\left(\tilde{P}_{t}-\lambda\right)^{-1}$,

where $W \in C_{0}^{\infty}(\hat{O}), V+W>\lambda_{0}$ on $\hat{O}, \tilde{P}_{t}$ denotes the operator $\tilde{P}=-h^{2} \Delta+V+W$ acting on $H\left(\Lambda_{t G},\langle\xi\rangle^{2}\right)$, and $E_{t}, E_{t}^{ \pm}$are defined in the following way: let $\Psi \in H\left(\Lambda_{t G}, 1\right)$ be the resonant state associated to $\varrho(h)$, normalized so that $\langle\Psi, \bar{\Psi}\rangle_{t}=1$, where $\langle\cdot, \cdot\rangle_{t}$ is the duality bracket between $H\left(\Lambda_{t G}, 1\right)$ and $H\left(\Lambda_{-t G}, 1\right)$ (which is a kind of extension of the $L^{2}$-bracket: cf. [He-Sj, Proposition (8.8)]. Let also $P_{t}^{\prime}$ be restriction of $P$ to $\left\{u \in H\left(\Lambda_{t G},\langle\xi\rangle^{2}\right),\langle u, \bar{\Psi}\rangle_{t}=0\right\}$. Then $P_{t}^{\prime}-\lambda$ is invertible [with values in $\left\{u \in H\left(\Lambda_{t G}, 1\right),\langle u, \bar{\Psi}\rangle_{t}=0\right\}$ ] and we have for any $v \in H\left(\Lambda_{t G}, 1\right)$ and $v^{+} \in \mathbb{C}$ :

$$
\begin{aligned}
E_{t}(\lambda) v & =\left(P_{t}^{\prime}-\lambda\right)^{-1}\left(v-\langle v, \bar{\Psi}\rangle_{t} \Psi\right), \\
E_{t}^{-} v & =\langle v, \bar{\Psi}\rangle_{t}, \\
E_{t}^{+} v^{+} & =v^{+} \Psi .
\end{aligned}
$$

Of course, we also have a similar formula for $R_{-t}(\lambda)$. Since $\lambda_{0}$ is a non-trapping energy for $\tilde{P}$, we know from a result of Robert and Tamura (Ro-Ta 1] (see also [Ge-Ma2] for a shorter proof of it) that for any $s>\frac{1}{2}$ :

$$
\left\|\langle x\rangle^{-s} \widetilde{R}(\lambda \pm i 0)\langle x\rangle^{-s}\right\|=O\left(h^{-1}\right),
$$

where $\tilde{R}$ denotes the resolvent of $\tilde{P}$.

If $\chi \in C_{0}^{\infty}\left(\mathbb{R}_{+}^{*}\right), \chi=1$ near $\lambda_{0}$, we know from the functional calculus of [He-Ro] that, if we set $\theta(t)=\frac{1-\chi(t)}{t-\lambda}$ for $\lambda$ close to $\lambda_{0}$, then

$$
(1-\chi(\widetilde{P})) \tilde{R}(\lambda \pm i 0)=\tilde{R}(\lambda \pm i 0)(1-\chi(\widetilde{P}))=\theta(\widetilde{P})
$$

is a bounded operator from $H^{m}\left(\mathbb{R}^{n)}\right.$ to $H^{m+2}\left(\mathbb{R}^{n}\right), \forall m \in \mathbb{R}$. Since moreover, for any $\alpha \in \mathbf{N}^{n}$ the operator $\langle x\rangle^{-s}\left(h \partial_{x}\right)^{\alpha} \chi(\tilde{P})\langle x\rangle^{s}$ is $O(1)$ on $L^{2}$, we deduce from (1.2) that:

$$
\left\|\langle x\rangle^{-s}\left(h \partial_{x}\right)^{\alpha} \tilde{R}(\lambda \pm i 0)\left(h \partial_{x}\right)^{\beta}\langle x\rangle^{-s}\right\|=O\left(h^{-1}\right)
$$

for any $\alpha, \beta \in \mathbb{N}^{n,}|\alpha|+|\beta| \leqq 2$.

From [Ge-Ma1, Lemma 1.1], we also know that $R_{t}(\hat{\lambda})$ coincides with $R(\lambda \pm i 0)$ on $C_{0}^{\infty}\left(\mathbb{R}^{n}\right)$. Thus, using (1.3) formula (1.1) implies, for any $K \Subset \Sigma$ :

$$
\begin{aligned}
\left\|1_{K}\left(h \partial_{x}\right)^{\alpha} R(\lambda+i 0)\left(h \partial_{x}\right)^{\beta} 1_{K}\right\|= & O\left(h^{-1}\right)+\frac{1}{|\lambda-\varrho(h)|} O\left(\operatorname{Sup}_{K}\left|(h \partial)^{\alpha} \Psi\right| \cdot\left|(h \partial)^{\beta} \Psi\right|\right) \\
& +\left\|1_{K}\left(h \hat{\partial}_{x}\right)^{\alpha} E_{t}(\lambda) W\left(\tilde{P}_{t}-\lambda\right)^{-1}\left(h \partial_{x}\right)^{\beta} 1_{K}\right\| \\
& +\left\|1_{K}\left(h \hat{\partial}_{x}\right)^{\alpha} E_{t}^{+} E_{t}^{-}\left(\tilde{P}_{t}-\lambda\right)^{-1}\left(h \partial_{x}\right)^{\beta} 1_{K}\right\| .
\end{aligned}
$$

From [Ge-Ma, formula (3.16)], we have:

$$
\left\|1_{K}\left(h \partial_{x}\right)^{\alpha} E_{t}(\lambda) W\right\|=O_{t}\left(e^{-\varepsilon_{0} / h}\right)
$$


with $\varepsilon_{0}>0$ independent of $t$, and, from [He-Sj, Theorem 9.9]:

$$
\forall \varepsilon>0, \quad \sup _{K} \sum_{|\alpha| \leqq 2}\left|\partial_{x}^{\alpha} \Psi\right|=O_{\varepsilon}\left(e^{-\left(S_{0}-\varepsilon\right) / h}\right) .
$$

Then, it remains to study the operator

$$
1_{K}\left(h \partial_{x}\right)^{\alpha} E_{t}^{+} E_{t}^{-}\left(\widetilde{P}_{t}-\lambda\right)^{-1}\left(h \partial_{x}\right)^{\beta} 1_{K} \cdot
$$

Its kernel is:

$$
K_{t}(x, y)=h^{|\alpha|+|\beta|} 1_{K}(x) 1_{K}(y) \partial_{x}^{\alpha} \Psi(x)\left[\partial_{x}^{\beta}\left(\tilde{P}_{-t}-\lambda\right)^{-1} \bar{\Psi}\right](y) .
$$

From the pseudo-differential calculus of $[\mathrm{He}-\mathrm{Sj}$, Sect.6], we know that $(h \partial)^{\beta} \cdot\left(\widetilde{P}_{-t}-\lambda\right)^{-1}$ is bounded on $H\left(\Lambda_{-t G}, 1\right)$ uniformly with respect to $h$.

Now let $M=\{y \in \hat{O} \mid d(y, \partial \hat{O})>\eta\}$ with $\eta>0$ small enough. Let also $\varphi$ be the normalized Dirichlet eigenfunction of $P$ on $L^{2}(M)$, associated to the eigenvalue closest to $\operatorname{Re} \varrho(h)$. We then see as in [Ge-Ma1, Sect. 3] that if $\chi \in C_{0}^{\infty}(M)$,

$$
\begin{gathered}
\chi=1 \text { on }\{y \in M \mid d(y, \partial M)>\eta\}, \text { then: } \\
\|\bar{\Psi}-\chi \varphi\|_{H(\Lambda-t G, 1)}=O_{t}\left(e^{-\varepsilon_{1} / h}\right)
\end{gathered}
$$

with $\varepsilon_{1}>0$ independant of $t$. (In fact, one can take $\varepsilon_{1}=S_{0}-\varepsilon(\eta)$ with

$$
\varepsilon(\eta) \rightarrow 0 \quad \text { as } \quad \eta \rightarrow 0 \text {.) }
$$

Thus, using (1.8), it follows from (1.7):

$$
\begin{aligned}
K_{t}(x, y)= & h^{|\alpha|+|\beta|} 1_{K}(x) 1_{K}(y) \partial_{x}^{\alpha} \Psi(x) \partial_{x}^{\beta} \tilde{R}(\lambda-i 0) \chi \varphi(y) \\
& +1_{K}(x) 1_{K}(y) \partial_{x}^{\alpha} \Psi(x) \mu_{t}(y)
\end{aligned}
$$

with

$$
\left\|\mu_{t}\right\|_{H(A-t G .1)}=O_{t}\left(e^{\left.-\varepsilon_{1} / h\right)} .\right.
$$

Finally, using Lemma 3.2 of [Ge-Ma1] and (1.2), (1.6), we deduce from (1.9):

$$
\text { \|I } K_{t} \|_{L^{2}\left(\mathbb{R}^{2 n}\right)}=O\left(e^{-\varepsilon_{2} / h}\right)
$$

with $\varepsilon_{2}>0$.

Putting (1.5), (1.6), and (1.10) in (1.4), this ends the proof of Lemma 1.2.

Proof of Proposition 1.1. We follow the strategy of [Ro-Ta1], and use the same notations as in the proof of Lemma 1.2. Let $J \in C^{\infty}\left(\mathbb{R}^{n} ; \mathbb{R}\right)$ with supp $J \subset \Sigma$, and $J=1$ outside a compact set.

For any $f \in C_{0}^{\infty}\left(\mathbb{R}^{n}\right)$ with $\operatorname{supp} f \subset K \Subset \Sigma$, let $v=J R(\lambda+i 0)(h \partial)^{\beta} f$, where $\beta \in \mathbb{N}^{n,}|\beta| \leqq 2$.

We then have:

$$
(\tilde{P}-\lambda) v=(P-\lambda) v=\left[-h^{2} \Delta, J\right] R(\lambda+i 0)(h \partial)^{\beta} f+J(h \partial)^{\beta} f .
$$

Using the fact that $v$ is outgoing (i.e. $v \in H\left(\Lambda_{t G}, 1\right)$ with $t>0$ ), we get:

$$
v=\widetilde{R}(\lambda+i 0)\left[-h^{2} \Delta, J\right] R(\lambda+i 0)(h \hat{\partial})^{\beta} f+\widetilde{R}(\lambda+i 0) J(h \partial)^{\beta} f .
$$


Thus, using (1.3) and Lemma 1.2, this gives the estimate:

$$
\left\|\langle x\rangle^{-s}(h \partial)^{\alpha} v\right\|_{L^{2}}=\left[O\left(h^{-1}\right)+O_{\varepsilon}\left(\frac{e^{-\left(2 S_{0}-\varepsilon\right) / h}}{\mid \lambda-\varrho \Phi h) \mid}\right)\right]\|f\|_{L^{2}}
$$

for any $K \Subset \Sigma$ and $|\alpha|+|\beta| \leqq 2$.

This proves that:

$$
\left\|\langle x\rangle^{-s}(h \partial)^{\alpha} J R(\lambda \pm i 0)(h \partial)^{\beta} 1_{K}\right\|=O\left(h^{-1}\right)+O_{\varepsilon}\left(\frac{e^{-\left(2 S_{0}-\varepsilon\right) / h}}{\mid \lambda-\varrho \Phi h) \mid}\right)
$$

for any $K \Subset \Sigma$ and $|\alpha|+|\beta| \leqq 2$.

Now we consider $v=J R(\lambda+i 0) f$, where

$$
f=\langle x\rangle^{-s} J(x)\left(h \partial_{x}\right)^{\beta} g, \quad g \in C_{0}^{\infty}\left(\mathbb{R}^{n}\right) .
$$

As before, we have:

$$
v=\widetilde{R}(\lambda+i 0)\left[-h^{2} \Delta, J\right] R(\lambda+i 0) f+\widetilde{R}(\lambda+i 0) J f
$$

and then, using (1.11) and (1.3), we get:

$$
\left\|\langle x\rangle^{-s}(h \partial)^{\alpha} v\right\|_{L^{2}}=\left[O\left(h^{-1}\right)+O_{\varepsilon}\left(\frac{e^{-\left(2 S_{0}-\varepsilon\right) / h}}{|\lambda-\varrho(h)|}\right)\right]\|g\|_{L^{2}}
$$

for any $\alpha \in \mathbb{N}^{n},|\alpha|+|\beta| \leqq 2$.

We have proved:

$$
\left\|J\langle x\rangle^{-s}(h \partial)^{\alpha} R(\lambda \pm i 0)(h \partial)^{\beta}\langle x\rangle^{-s} J\right\|_{\mathscr{L}\left(L^{2}, L^{2}\right)}=O\left(h^{-1}\right)+O_{\varepsilon}\left(\frac{e^{-\left(2 S_{0}-\varepsilon\right) / h}}{|\lambda-\varrho(h)|}\right) .
$$

Summing up the information given by Lemma 1.2,(1.11), and (1.12), the result of Proposition 1.1 follows easily.

For ending this section, let us now recall the main result of [Ge-Ma, Sect. 3] which will be used afterwards.

Let $\frac{\partial e}{\partial \lambda}$ be the spectral function of $P$, i.e. the kernel of $\frac{\partial E}{\partial \lambda}$, where $d E_{\lambda}=\frac{\partial E}{\partial \lambda} d \lambda$ is the spectral resolution of $P$ on $\{\lambda>0\}$.

We also denote $d$ the Agmon distance associated to the degenerate metric $\left(V(x)-\lambda_{0}\right)_{+} d x^{2}$, and we define:

$$
\tilde{d}(x, y)=\min \left(S_{0}+d(x, U)+d(y, \partial \hat{O}), S_{0}+d(y, U)+d(x, \partial \hat{O})\right)
$$

where, as before, $S_{0}=d(U, \partial \hat{O})$. $\widetilde{d}(x, y)$ can be seen as the "touristic distance" necessary to go from $x$ to $y$ visiting the well $U$ and the sea $\Sigma=\mathbb{R}^{n} \backslash \hat{O}$. [Note that $\widetilde{d}(x, y) \geqq S_{0}$ for any $x, y$.]

$\widetilde{P}$ is defined as in the proof of Lemma 1.2, with

$$
w \in C_{0}^{\infty}(\{d(x, U)<\eta\}) \quad(\eta>0 \text { small enough }) .
$$


Theorem 1.3 [Ge-Ma1. Theorem 3.1]. Under assumptions (H1) to (H4), the spectral function $\frac{\partial e}{\partial \lambda}$ of $P$ is related to the spectral function $\frac{\partial \tilde{e}}{\partial \lambda}$ of $\tilde{P}$ by the formula:

$$
\frac{\partial e}{\partial \lambda}(x, y, \lambda, h)=\frac{\partial \tilde{e}}{\partial \lambda}(x, y, \lambda, h)-\frac{1}{\pi} \operatorname{Im}\left[(\lambda-\varrho(h))^{-1} \Psi(x) \Psi(y)\right]+K(x, y, \lambda, h),
$$

where $K$ satisfies: $\forall \alpha, \beta \in \mathbb{N}^{n}$

$$
\partial_{x}^{\alpha} \partial_{y}^{\beta} K(x, y, \lambda, h)=O_{\eta}\left(e^{-(\alpha(x, y)-\varepsilon(\eta)) / h}\right)
$$

locally uniformly for $(x, y) \in \mathbb{R}^{2 n}$, and uniformly for $\lambda \in I(h), h>0$ small enough.

Here $\varepsilon(\eta) \rightarrow 0$ as $\eta \rightarrow 0$.

\section{Breit-Wigner Formula for the Scattering Phase}

Recall from the introduction that the derivative $\frac{d s}{d \lambda}$ of the scattering phase is related to the spectral function $\frac{\partial e}{\partial \lambda}$ by:

$$
\frac{d s}{d \lambda}(\lambda, h)=\frac{1}{2 \lambda} \int_{\mathbb{R}^{n}}(2 V(x)+x \cdot \nabla V(x)) \frac{\partial e}{\partial \lambda}(x, x, \lambda, h) d x .
$$

Moreover, the scattering phase is well defined if the rate of decay of $V$ appearing in $(\mathrm{H} 2)$ satisfies $\varrho>n$. Here, we shall need a stronger assumption, namely:

$$
\varrho>n+1 .
$$

Then, our result is:

Theorem 2.1. Under assumption (H1) to (H4) and (2.2), one has, for any $\varepsilon>0$ :

$$
\frac{d s}{d \lambda}(\lambda, h)=\frac{1}{\pi} \frac{\operatorname{Re} \varrho(h)}{\lambda} \operatorname{Im}\left(\frac{1}{\varrho(h)-\lambda}\right)+O_{\varepsilon}\left(\frac{e^{-\left(2 S_{0}-\varepsilon\right) / h}}{|\varrho(h)-\lambda|}\right)+O\left(h^{-n}\right)
$$

uniformly for $\lambda \in I(h), h>0$ small enough.

Remark 2.2. Note that, by the results of [He-Sj] or [C-D-K-S], the function $\lambda \rightarrow \operatorname{Im} \frac{1}{\varrho(h)-\lambda}$ presents a spike at $\lambda=\operatorname{Re} \varrho(h)$ of height $|\operatorname{Im} \varrho(h)|^{-1} \geqq \frac{1}{C_{\varepsilon}}$ - $e^{\left(2 S_{0}-\varepsilon\right) / h}$ for any $\varepsilon>0$ (with $C_{\varepsilon}>0$ ). Thus, Theorem 2.1 will provide in many cases the behaviour of $\frac{d s}{d \lambda}$ for $\lambda$ near $\operatorname{Re} \varrho(h)$.

Before proving Theorem 2.1, let us give an easy application of it, concerning the increases of the phase shift around the real part of a resonance.

Corollary 2.3. Under assumptions of Theorem 2.l, for any $\delta(h)>0$ such that:

i) $\operatorname{Re} \varrho(h) \pm \delta(h) \in I(h)$ for any $h>0$ small enough.

ii) $h^{-n} \delta(h) \rightarrow 0$ and $\delta(h)|\operatorname{Im} \varrho(h)|^{-1} \rightarrow+\infty$ as $h \rightarrow 0$ we have the following result: the increases of the phase-shift:

$$
\Delta_{ \pm} s(h):= \pm(s(\operatorname{Re} \varrho(h) \pm \delta(h), h) \mp s(\operatorname{Re} \varrho(h), h)
$$

satisfy: $\lim _{h \rightarrow 0} \Delta_{ \pm} s(h)=1 / 2$. 
Remark 2.4. In particular, the phase shift keeps positive for $h$ small enough. Then, we see that this kind of shape resonance agrees with a physical notion of resonance related to the time-delay: see for example the book of Newton [Ne, Chaps. 11 and 12]. The fact that the semiclassical limit is $1 / 2$ also agrees with the well-known physical interpretation of a resonance [Ne, p. 316].

Proof of Theorem 2.1. We apply formula (2.1) and Theorem 1.3. Since $\frac{\partial \tilde{e}}{\partial \lambda}$ is $\mathcal{O}\left(h^{-n}\right)$ locally uniformly (cf. [Ro-Ta2; Ge-Ma1]), we then obtain:

$$
\begin{aligned}
\frac{d s}{d \lambda}(\lambda, h)= & \frac{1}{2 \pi \lambda} \operatorname{Im} \frac{1}{\varrho(h)-\lambda}\left[\int_{O}(\Psi(x))^{2}(2 V(x)+x \nabla V(x)) d x\right] \\
& \ldots+O_{\varepsilon}\left(e^{-\left(2 S_{0}-\varepsilon\right) / h}\right)+O\left(h^{-n}\right)+r(\lambda, h),
\end{aligned}
$$

with

$$
r(\lambda, h)=\frac{1}{2 \lambda} \int_{\Sigma}(2 V(x)+x \nabla V(x)) \frac{\partial e}{\partial \lambda}(x, x, \lambda, h) d x,
$$

where, as before, $\Sigma=\mathbb{R}^{n} \backslash \hat{O}$ is the sea.

At first, let us examine the first term appearing in (2.3). Let

$$
A=\frac{1}{2 i}(x \cdot \nabla+\nabla \cdot x) \text {. }
$$

We then have the identity between differential operators:

$$
2 V+x \cdot \nabla V=2 P+\frac{1}{i}[P, A] .
$$

Moreover, we know that $\Psi \in C^{\infty}\left(\mathbb{R}^{n}\right), P \Psi=\varrho(h) \Psi$, and $\forall \alpha \in \mathbb{N}^{n,} \forall \varepsilon>0$, $\partial^{\alpha} \Psi(x)=O_{\varepsilon, \alpha}\left(e^{-(d(x, U)-\varepsilon) / h)}\right)$ locally uniformly in $x$ (cf. [He-Sj]).

Moreover $\int_{O}(\Psi(x))^{2} d x=1+\mathcal{O}_{\varepsilon}\left(e^{-\left(2 S_{0}-\varepsilon\right) / h}\right)$. Thus, using (2.5) and Green's formula (possibly after having changed a little bit $\hat{\partial} \hat{O}$ so that is becomes smooth enough), it is easy to see that for any $\varepsilon>0$ :

$$
\int_{O}(\Psi(x))^{2}(2 V(x)+x \nabla V(x)) d x=2 \varrho(h)+O_{\varepsilon}\left(e^{-\left(2 S_{0}-\varepsilon\right) / h}\right),
$$

so that we get, using also the fact that $\varrho(h)$ and $\operatorname{Re} \varrho(h)$ only differ from a $O_{\varepsilon}\left(h^{-\left(2 S_{0}-\varepsilon\right) / h}\right)$ :

$\frac{d s}{d \lambda}(\lambda, h)=\frac{1}{\pi} \frac{\operatorname{Re} \varrho(h)}{\lambda} \operatorname{Im}\left(\frac{1}{\varrho(h)-\lambda}\right)\left(1+O_{\varepsilon}\left(e^{-\left(2 S_{0}-\varepsilon\right) / h}\right)\right)+O\left(h^{-n}\right)+r(\lambda, h)$.

Then, it remains to estimate $r(\lambda, h)$. By the properties of $\frac{\partial e}{\partial \lambda}$ given in Theorem 1.3, it is enough to estimate:

$$
r_{1}(\lambda, h)=\int J(x)(2 V(x)+x \nabla V(x)) \frac{\partial e}{\partial \lambda}(x, x, \lambda, h) d x,
$$

where $J \in C^{\infty}\left(\mathbb{R}^{n}\right), \operatorname{supp} J \subset \Sigma, J=1$ outside a compact set.

Moreover, since $2 V(x)+x \nabla V(x)=O\left(\langle x\rangle^{-g}\right)$ uniformly in $\mathbb{R}^{n}$, and $\varrho>n+1$, it is enough to prove: 
Lemma 2.5. Under assumptions (H1) to $(\mathrm{H} 4)$, we have for any $s>\frac{1}{2}$ and $\varepsilon>0$ :

$$
J(x)\langle x\rangle^{-s} \frac{\partial e}{\partial \lambda}(x, y, \lambda, h)\langle y\rangle^{-s} J(y)=O_{\varepsilon}\left(h^{1-n-\varepsilon}\right)+O_{\varepsilon}\left(\frac{e^{-\left(2 S_{0}-\varepsilon\right) / h}}{|\hat{\lambda}-\varrho(h)|}\right)
$$

uniformly for $(x, y) \in \mathbb{R}^{2 n,} \lambda \in I(h), h>0$ small enough.

Proof. Let $Q_{\lambda}=J(x)\langle x\rangle^{-s} \frac{\partial E}{\partial \lambda}\langle x\rangle^{-s} J(x)$.

Since $\frac{\partial E}{\partial \lambda}=\frac{1}{2 i \pi}(R(\lambda+i 0)-R(\lambda-i 0))$, we have by Proposition 1.1:

$$
\left\|Q_{\lambda}\right\|_{\mathscr{L}\left(L^{2}, H^{2}\right)}=O\left(h^{-1}\right)+O_{\varepsilon}\left(\frac{e^{-\left(2 S_{0}-\varepsilon\right) / h}}{|\lambda-\varrho(h)|}\right) .
$$

Moreover, since $(P-\lambda) \frac{\partial E}{\partial \lambda}=0$ on $L_{s}^{2}$, we have for any $c>0$ :

$$
(P+C) Q_{\lambda}=\left[P, J\langle x\rangle^{-s}\right] \frac{\partial E}{\partial \lambda}\langle x\rangle^{-s} J+(C+\lambda) Q_{\lambda}
$$

So that, by (1.10), we see that $(P+C) Q_{\lambda}$ satisfies the same kind of estimate as $Q_{\lambda}$ in (2.8). Iterating the procedure, we find that for any $k \in \mathbb{N}$ :

$$
\left\|(P+C)^{k} Q_{\lambda}\right\|_{\mathscr{L}\left(L^{2}, H^{2}\right)}=O\left(h^{-1}\right)+O_{\varepsilon}\left(+\frac{e^{-\left(2 S_{0}-\varepsilon\right) / h}}{|\lambda-\varrho(h)|}\right)
$$

uniformly for $h>0$ small enough.

By an easy estimate, we also know that if we take $C>0$ large enough, then $(P+C)^{-1}$ is $O\left(h^{-2}\right)$ from $H^{m}\left(\mathbb{R}^{n}\right)$ to $H^{m+2}\left(\mathbb{R}^{n}\right)$ for any $m \in \mathbb{R} \doteq$ Thus (2.9) implies:

$$
\left\|Q_{\lambda}\right\|_{\mathscr{L}\left(L^{2}, H^{2 k}\right)}=O\left(h^{1-2 k}\right)+O_{\varepsilon}\left(\frac{e^{-\left(2 S_{0}-\varepsilon\right) / h}}{|\hat{\lambda}-\varrho(h)|}\right)
$$

for any $k \geqq 0$. Then, taking the adjoint, (2.10) is also true in the norm of $\mathscr{L}\left(H^{-2 k}, L^{2}\right)$, and applying again $(P+C)^{k}$ to $Q_{\lambda}$, we conclude from this:

$$
\left\|Q_{\lambda}\right\|_{\mathscr{L}\left(H^{-2 k}, H^{2 k}\right)}=O\left(h^{1-4 k}\right)+O_{\varepsilon}\left(\frac{e^{-\left(2 S_{0}-\varepsilon\right) / h}}{|\lambda-\varrho(h)|}\right) .
$$

In particular, if we take $k>\frac{n}{4}$ and apply $Q_{\lambda}$ to the Dirac measure $\delta_{y_{0}}\left(y_{0} \in \mathbb{R}^{n}\right.$ arbitrary), and then if we make the $H^{2 k}-H^{-2 k}$ duality product between $Q_{\lambda}\left(\delta_{y_{0}}\right)$ and $\delta_{x_{0}}\left(x_{0} \in \mathbb{R}^{n}\right.$ arbitrary), we find for the kernel $q_{\lambda}$ of $Q_{\lambda}$ (using also the fact that $\left\|\delta_{y_{0}}\right\|_{H^{-2 k}}$ is independent of $\left.y_{0} \in \mathbb{R}^{n}\right)$ :

$$
q_{\lambda}\left(x_{0}, y_{0}\right)=\mathcal{O}\left(h^{1-4 k}\right)+\mathcal{O}_{\varepsilon}\left(\frac{e^{-\left(2 S_{0}-\varepsilon\right) / h}}{|\lambda-\varrho(h)|}\right)
$$

uniformly for $x_{0}, y_{0}$ in $\mathbb{R}^{n}$ which is the wanted result.

Proof of Corollary 2.3. Let $\alpha(h)=\operatorname{Re} \varrho(h)$ and $\beta(h)=-\operatorname{Im} \varrho(h)(>0)$. Then we have:

$$
\Delta_{+} s(h)=\int_{\alpha(h)}^{\alpha(h)+\delta(h)} \frac{d s}{d \lambda}(\lambda, h) d \lambda,
$$


using Theorem 2.1 and the fact that $\alpha(h)+\delta(h)$ tend to $\lambda_{0}$ as $h$ tends to 0 , we get:

$$
\begin{aligned}
\Delta S_{+}(h)= & \frac{1}{\pi} \int_{\alpha(h)}^{\alpha(h)+\delta(h)} \frac{\beta(h)}{(\lambda-\alpha(h))^{2}+\beta(h)^{2}}(1+O(1)) d \lambda \\
& +O\left(e^{-\left(2 S_{0}-\varepsilon\right) / h}\right) \int_{\alpha(h)}^{\alpha(h)+\delta(h)} \frac{d \lambda}{\sqrt{(\lambda-\alpha(h))^{2}+\beta(h)^{2}}} \\
& +O\left(\delta(h) h^{-n}\right),
\end{aligned}
$$

and thus:

$$
\begin{aligned}
\Delta_{+} s(h) & =\frac{1}{\pi}\left[\tan ^{-1} \frac{\delta(h)}{\beta(h)}\right](1+o(1))+O\left(e^{-\left(2 S_{0}-\varepsilon\right) / h}\right) \ln (\delta(h) \\
& +\sqrt{\left.\delta(h)^{2}+\beta(h)^{2}\right)}+o(1),
\end{aligned}
$$

which tends to $1 / 2$ as $h$ tends to 0 . By the same argument we also have:

$$
\lim _{h \rightarrow 0} \Delta_{-} s(h)=\frac{1}{2} \text {. }
$$

Remark 2.6. Let $J \in C^{\infty}\left(\mathbb{R}^{n}\right)$, supp $J \subset \Sigma, J=1$ outside a compact set. Then, if we set

$$
\Phi(x, \lambda, \omega, h)=[\mathbb{1}-R(\lambda+i 0)(P-\lambda)]\left(J(x) e^{i \sqrt{x x} \omega / h}\right)
$$

it is well known that we have (see e.g. [Is])

$$
\frac{\partial e}{\partial \lambda}(x, x, \lambda, h)=(2 \pi h)^{-n} \lambda^{\frac{n-2}{2}} \int_{S^{n-1}}|\Phi(x, \lambda, \omega, h)|^{2} d \omega .
$$

Thus, an alternative method to estimate $r(\lambda, h)$ defined in (2.4) would be to use (2.12) and Proposition 1.1. In that case, it is sufficient to assume $\varrho>n$. Nevertheless, because of the presence of the square of $|\Phi|$ appearing in (2.12), this leds to an error term of order $e^{-\left(4 S_{0}-\xi\right) / h}|\lambda-\varrho(h)|^{-2}$. Then, this kind of estimate can be useful only in cases where one can also get a sufficient lower bound of $\operatorname{Im} \varrho(h)$. (For instance when $U$ is pointwise and non-degenerate, Theorem 10.16 of [He-Sj] describes a situation where Theorem 2.1 is still valid with $n<\varrho \leqq n+1$.)

\section{The Total Scattering Cross-Section}

We have seen in the introduction that the total scattering cross-section $\sigma$ is given by:

$\sigma(\omega, \lambda, h)=\pi \lambda^{-1 / 2} \cdot h^{-1} \iint \frac{\partial e}{\partial \lambda}(x, y, \lambda, h) V(x) V(y) e^{i \sqrt{\lambda}(x-y) \cdot \omega / h} d x d y$.

Here, we assume that the index decay $\varrho$ satisfies:

$$
\varrho>\frac{n+1}{2},
$$

which implies that $\langle x\rangle^{s} V \in L^{2}\left(\mathbb{R}^{n}\right)$ for $s>\frac{1}{2}, s-\frac{1}{2}$ small enough. 
We then have:

Theorem 3.1. Under assumptions $(\mathrm{H} 1)$ to $(\mathrm{H} 4)$ with $\varrho>\frac{n+1}{2}$, we have for any $\varepsilon>0$ :

$$
\begin{aligned}
\sigma(\omega, \lambda, h)= & \frac{1}{\pi} \operatorname{Im} \frac{1}{\varrho(h)-\lambda}\left|\int_{O} \Psi(x) V(x) e^{i \sqrt{\lambda} \omega \cdot x / h} d x\right|^{2} \\
& +O_{\varepsilon}\left(\frac{e^{-\left(S_{0}-\varepsilon\right) / h}}{|\hat{\lambda}-\varrho(h)|}\right)+O\left(h^{-n}\right) .
\end{aligned}
$$

Remark 3.2. This result can probably also be deduced from the results from Nakamura [Na]. However, since the methods are quite different, we give here an independent proof of it.

Proof of Theorem 3.1. First of all, we remark that, by the same kind of proof as in Proposition 1.1, we also have:

$$
\left\|1_{\Sigma}\langle x\rangle^{-s}\left(h \partial_{x}\right)^{\alpha} R(\lambda \pm i 0)\left(h \partial_{x}\right)^{\beta}\langle x\rangle^{-s}\right\|=O\left(h^{-1}\right)+O_{\varepsilon}\left(\frac{e^{-\left(S_{0}-\varepsilon\right) / h}}{|\lambda-\varrho(h)|}\right)
$$

for any $\varepsilon>0, s>\frac{1}{2}$, and $|\alpha|+|\beta| \leqq 2$. In fact, the only difference comes from (1.4) where we have now to estimate $\left\|1_{K_{1}}\left(h \partial_{x}\right)^{\alpha} R(\hat{\lambda} \pm i 0)\left(h \hat{\partial}_{x}\right)^{\beta} 1_{K_{2}}\right\|$ with $K_{1} \Subset \Sigma$, $K_{2} \Subset \mathbb{R}^{n}$. To do this, we use the fact that

$$
\sup _{K_{2}} \sum_{|x| \leqq 2}\left|\partial^{\alpha} \Psi\right|=O_{\varepsilon}\left(e^{\varepsilon / h}\right)
$$

for any $\varepsilon>0$ and $\alpha \in N^{n}$.

Then, using Theorem 1.2, we obtain the same bound as in (3.5) with $2 S_{0}$ replaced by $S_{0}$. As in Proposition 1.1, we then show that

$$
\left\|1_{\Sigma}\langle x\rangle^{-s} \frac{\partial E}{\partial \hat{\lambda}} 1_{K_{2}}\right\|_{\mathscr{L}\left(L^{2} \cdot L^{2}\right)}=O\left(h^{-1}\right)+O_{\varepsilon}\left(\frac{e^{-\left(S_{0}-\varepsilon\right) / h}}{|\lambda-Q(h)|}\right),
$$

which, added to the result of Proposition 1.1, permits us to deduce directly (3.3).

Now, by (3.1) and Theorem 1.2, we see that for proving Theorem 3.1, it is enough to estimate:

$$
r(\omega, \lambda, h)=\int_{\Sigma \times R^{n}} \frac{\hat{\partial} e}{\partial \hat{\lambda}}(x, y, \hat{\lambda}, h) V(x) V(y) e^{i V \hat{\lambda}(x-y) \omega / h} d x d y,
$$

as well as the same expression but integrating on $R^{n} \times \sum$.

For any $s>\frac{1}{2}$, we have:

$$
r(\omega, \lambda, h)=\left\langle 1_{\Sigma}\langle x\rangle^{-s} \frac{\partial E}{\partial \lambda} V e^{-i \sqrt{\lambda} x \omega / h},\langle x\rangle^{s} V e^{-i \sqrt{\lambda} x \cdot \omega / h}\right\rangle_{L^{2}\left(R_{x}^{n}\right)},
$$

so that, using (3.3), we get:

$$
|r(\omega, \lambda, h)|=\left(O\left(h^{-1}\right)+O_{\varepsilon}\left(\frac{e^{-\left(S_{0}-\varepsilon\right) / h}}{|\lambda-\varrho(h)|}\right)\right)\left\|\langle x\rangle^{s} V\right\|_{L^{2}}^{2} .
$$


Taking $s$ close enough to $\frac{1}{2}$ so that $\langle x\rangle^{s} V \in L^{2}\left(R^{n}\right)$, and proving similarly the same estimate when integrating on $R^{n} \times \Sigma$, end the proof of Theorem 3.1.

\section{References}

[Ag-Co] Aguilar, J., Combes, J.M.: A class of analytic perturbations for one body Schrödinger Hamiltonians. Commun. Math. Phys., 22, 269-279 (1971)

[Bi-Kr] Birman, M.S., Krein, M.G.: On the theory of wave operators and scattering operators. Dokl. Akad. Nauk SSSR 144, 475-478 (1962), (in Russian)

[CdV] Colin de Verdière, Y.: Une formule de trace pour l'opérateur de Schrödinger dans $R^{3}$. Ann. Sci. Ec. Norm. Sup. 14, $27-39$ (1981)

[C-D-K-S] Combes, J.M., Duclos, P., Klein, M., Seiler, R.: The shape resonance. Commun. Math. Phys. 110, 215-236 (1987)

[En-Si] Enss, V., Simon, B.: Total cross sections in non-relativistic scattering theory, quantum mechanics in mathematics, chemistry and physics. Gustafson K. E. (ed.). In: P. Reinhart. New York: Plenum Press 1981

[Ge-Ma] Gérard, C., Martinez, A.: [1] Semiclassical asymptotics for the spectral function of long range Schrödinger operators. J. Funct. Anal. (to appear)

[2] Principe d'absorption limite pour des opérateurs de Schrödinger à longue portée. C. R. Acad. Sci. Paris, 306, Sér. I, 121-123 (1988)

[Gu] Guillopé, L.: Assymptotique de la phase de diffusion pour l'opérateur de Schrödinger avec potentiel. C.R. Acad. Sci. Paris 293, 601-603 (1981)

[He-Ma] Helffer, B., Martinez, A.: Comparaison entre les diverses notions de résonances. Helv. Phys. Acta, 60, 992-1003 (1987)

[He-Ro] Helffer, B., Robert, D.: Calcul fonctionnel par la transformation de Mellin et opérateurs admissibles. J. Funct. Anal. 53, 246-268 (1983)

[He-Sj] Helffer, B., Sjöstrand, J.: Résonances en limite semi-classique. Bull. S.M.F., mémoire n $24 / 25$, tome 114 (1986)

[Hi-Si] Hislop. P., Sigal, M.: Shape resonances in quantum mechanics, Proceedings of the International Conferences on Diff. Eq. In: Math. Phys., Birmingham (Alabama), March 1986

[Is] Isozaki, H.: Differentiability of generalized Fourier Transforms associated with Schrödinger Operators. J. Math. Kyoto Univ. 25 789-806 (1985)

[Iv-Sh] Ivrii, V.J., Shubin, M.A.: On the asymptotics of the spectral shift function. Dok1. Acad. Nauk. SSSR 263 (1982); Sov. Math. Dokl. 25

[Je-Ka] Jensen, A., Kato, T.: Asymptotic behavior of the scattering phase for exterior domains. Commun. P.D.E. 3, 1165-1195 (1978)

[La-Li] Landau. L., Lifchitz, E.: Mécanique quantique, théoric non relativiste. Editions Mir, Moscou (1967)

[Maj-Ra] Majda, A., Ralston, J.: An analogue of Weyl's theorem for unbounded domains. I, II, and III. Duke Math. J. 45, 183-196 (1978); 45, 513-536 (1978); 46, 725-731 (1979)

[Na] Nakamura, S.: Scattering theory for the shape resonance model, I-Non-resonant energies. II. Resonance scattering. Preprint Tokyo University

[Ne] Newton, R. G.: Scattering theory of waves and particles, Texts and monographs in physics, 2 nd éd. Berlin, Heidelberg, New York: Springer

[Pe-Po] Petkov, V., Popov, G.: Asymptotic behavior of the scattering phase for nontrapping obstacles. Ann. Inst. Fourier Grenoble 32, 111-149 (1982)

[Re-Si] Reed, M., Simon, B.: Methods of modern mathematical physics. New York: Academic Press 1978 
[Ro-Ta] Robert, D., Tamura, H.: [1] Semiclassical estimates for resolvents and asymptotics for total scattering cross-sections. Ann. I.H.P. 46, 415-442 (1987)

[2] Semiclassical asymptotics for local spectral densities and time delay problems in scattering processes. J. Funct Anal. 80, 124-147 (1988)

[So-Ya] Sobolev, A.V., Yafaev, D.R.: On the quasi-classical limit of the total scattering cross-section in non relativistic quantum mechanics. Ann. I.H.P. 44, 195-210 (1986)

[Ya] Yajima, K.: The quasi-classical limit of scattering amplitude $-L^{2}$ approach for short range potential. Jpn. J. Math. 13, 77-126 (1987)

Communicated by B. Simon

Received July 29, 1988; in revised form October 13, 1988 